\title{
Une mise en mots de la formation pour adultes
}

\begin{abstract}
Résumé
En se référant aux transformations des enjeux sociaux qui touchent le domaine de la formation des adultes la contribution pose deux questions : avec quels outils conceptuels la formation continue permet-elle de penser le devenir problématique des adultes dans le contexte postmoderne ? De quelles garanties de sens s'entoure ce devenir pour contourner les effets de crise, qui discréditent la mise en perspective d'un avenir à construire ?

Larticle met en évidence les défis qui attendent les pratiques de formation et donne des propositions de developpement. Les transformations touchent une reconfiguration de la formation et des sens qui lui sont habituellement conférés.
\end{abstract}

Les mots clés : formation des adultes, discours, adulte, injonction d'individualité, formation formelle, formation informelle.

\section{Adult Education: Conceptual Tools}

\begin{abstract}
Referring to the transformations and social conditions that affect also the field of adult education, this text raises the following questions: By what conceptual tools, in the current postmodern context, can adult education develop its issues? What does it mean today to be an adult learner? In what form of education?

The text analyzes the challenges that may influence the practice of education and indicates development proposals. The transformations concern the reconfiguration in the field of education and the meanings that are commonly assigned to it.
\end{abstract}

Keywords: adult education, discourse, adult, requirement of individuality, formal education, non-formal education.

* L'Université catholique de l'Ouest (Institut de psychologie et sociologie apliquées), France. 


\section{Une formation au service de quel adulte?}

Il n'est pas question ici de faire une généalogie du terme d'adulte ${ }^{1}$ et de ses transformations sémantiques successives dans notre langue française mais de nous interroger sur ces adultes in vivo que nous connaissons actuellement par rapport à ceux, pas si éloignés dans nos souvenirs, que nous avons connus dans notre enfance, notre mère ou notre père, nos grands-parents, des oncles et tantes ou grands oncles et grands-tantes, des voisines ou des voisins: les uns et les autres nous ont montré ou nous montrent encore des profils bien différents en fonction des époques où ils ont vécu. Ainsi, de ces cinquante dernières années, on peut identifier facilement et pour le moins quatre profils contrastés d'adultes, qui ne nous sont pas étrangers mais qui entretiennent vis-à-vis de l'instance formation des relations bien typées (Boutinet 2013) :

- l'adulte étalon est cet adulte considéré comme mature servant de référence, de norme pour les autres âges de la vie; il est caractéristique des générations adultes des années 1950, comme en son temps l'a bien mis en évidence Paul A. Osterrieth (1966) ; l'idéal de cet adulte, considéré comme une grande personne, est dans la régulation, l'équilibration de ses choix et décisions, de ses modes de vie; cet adulte voit dans le travail un métier à exercer avec un souci de continuité à travers son savoir-faire et sa stabilité ; il épouse le mode de vie traditionnel valorisant l'importance de la transmission intergénérationnelle: s'appuyant sur les acquis de sa formation initiale, sa formation continue, ne peut être que très occasionnelle, l'essentiel de ses apprentissages se déployant dans l'informel, lié à l'expérience qu'il tire des situations et des évènements vécus ;

- l'adulte en maturation ou en devenir s'institue en perspective (Rogers 1961), de ce fait en rupture avec le modèle précédent qu'il conteste (Lapassade 1963); il va caractériser les années 1960, voire 1970 ; cet adulte connaîtra une plus longue scolarisation que son prédécesseur, une scolarisation qu'il va vouloir bien souvent prolonger par la suite en participant à des actions de formation continue, en plein développement. C'est un adulte qui envisage le travail sous l'angle promotionnel; quittant délibérément la sphère des métiers, il entend faire de sa vie professionnelle une carrière (Holland 1973) avec ses mobilités qu'il va exploiter de façon à ce que cette carrière soit ascentionnelle (Bujold, Gingras 2000); dans ce contexte la formation continue à laquelle cet adulte pourra recourir de temps à autre va jouer le double rôle de mobilité et de perfectionnement ;

- l'adulte immature des années 1980-1990 s'installera à son tour en rupture avec le modèle qui l'a précédé de l'adulte en perspective, mais cette fois-ci

\footnotetext{
1 On pourra pour cette généalogie consulter notre travail L'immaturité de la vie adulte, Paris 1998, Presses Universitaire de France.
} 
malgré lui, car cet adulte devra assumer ou au moins craindre au cours de son itinéraire professionnel les méfaits de la situation de crise qui surprend dans les années 1975 la société postindustrielle avec un déficit de croissance économique et la montée d'un chômage endémique. Pour cet adulte, le principal souci dans son travail c'est d'avoir ou de pouvoir garder un emploi. Son immaturité vient de sa fragilité, de la peur de se retrouver dans une privation d'emploi (Boutinet 1999); ce dernier terme constitue désormais le maître mot pour qualifier le travail; dans ce contexte la formation va être à dominante conjoncturelle, entrevue comme le remède, l'un des moyens pour garder, retrouver ou consolider un emploi ;

- l'adule pluriel des années 2000-2010 a pris acte qu'il ne peut pas demeurer dans une crainte continuelle de perdre son emploi; il cherche donc à se donner des stratégies offensives pour s'en sortir de façon à éviter les formes de marginalisation qu'il peut observer autour de lui (Boulte 1995) ; il va donc jouer sur les pluralités (Lahire 1998): pluralité des compétences acquises, pluralité des emplois exercés, pluralité des transitions à aménager dans le cours d'une vie professionnelle, pluralité aussi des modes de vies conjugales et familiales. La formation sollicitée dans ce contexte se veut transitionnelle pour aider à aménager le passage entre une situation professionnelle qui ne peut perdurer dans son état actuel et celle qui va suivre, qu'il s'agit d'anticiper. Le cas échéant, lorsque les transitions seront difficiles à assumer (Houde 1999), l'entrée en formation sera remplacée par une démarche d'accompagnement, sollicitée par l'adulte lui-même.

A côté de cette dimension socio-historique pour caractériser les postures de l'adulte qui part en formation, d'autres paramètres seraient à prendre en compte, pour le moins trois autres paramètres particuliers et un paramètre tout à fait singulier. Au titre des paramètres particuliers, il est évident que le genre joue un rôle important selon que l'adulte femme envisage son travail au regard de ses contraintes familiales, selon que par exemple l'adulte homme entend ou pas valoriser son degré de mobilité. Mais ce paramètre du genre est à combiner avec deux autres paramètres, celui de l'avancée en âge et celui du niveau de formation initiale atteint: le jeune adulte n'a pas les mêmes demandes de formation que le sénior, ni la jeune mère de famille par rapport à la cadre du mitan de la vie ; selon par ailleurs qu'homme ou femme, l'adulte ait un niveau II ou I de formation au lieu de IV ou V ne le fera pas émettre les mêmes demandes de formation.

Enfin, paramètre tout à fait singulier susceptible de préformer les demandes de formation ${ }^{2}$, la dynamique du parcours individuel dans ce qui fait son originalité avec ses réalisations, ses aspirations, ses réussites et échecs va générer une forme d'engagement en formation ou de refus à s'engager, notamment si le passé scolaire

\footnotetext{
2 Désormais dans la suite du texte, chaque fois que nous utiliserons le terme de formation, ce sera pour désigner la formation continue pour adultes, excluant donc toute référence à la formation initiale mais évitant la redondance de rappeler chaque fois continue pour adultes.
} 
de l'adulte a été déjà parsemé d'échecs. Un entretien préalable à la formation avec l'adulte demandeur permettra de faire expliciter cette singularité du parcours décrit pour une prise de décision appropriée qui tienne compte de cette singularité, telle qu'elle a été verbalisée.

\section{De la formation promotionnelle à la formation réparatrice}

Ce demi-siècle qui vient de s'écouler a été marqué par un contraste très fort entre une période de croissance qui met en place la formation professionnelle continue et une période de crises renouvelées qui doit la faire subsister, comme le remarque Claude Dubar (1985) ; la première période est née avec les espoirs suscités par l'ambition promotionnelle de la formation continue à son origine c'est-à-dire au début des années 1970 ; elle va durer une dizaine d'années jusqu'au début des années 1980 ; une seconde période qui depuis occupe toujours le terrain, de 1980 à nos jours se veut réparatrice des déconvenues et échecs rencontrés par les adultes dans leur vie professionnelle ou personnelle, les uns et les autres liés à des contextes de vie plus difficiles et incertains qu'auparavant. Cette seconde période ne gomme pas toute possibilité de dispositif de formation à visée promotionnelle, les bilans de compétences et la validation des acquis de l'expérience peuvent en témoigner, mais ce type de formation devient minoritaire.

D'une période à l'autre le changement de décor amène la formation à devoir être pensée dans une diversité de situations, incluant celles qui touchent aux désillusions de la vie adulte; à ce propos le développement quelque peu spectaculaire au cours des vingt dernières années de l'éducation thérapeutique du patient pour les malades souffrant d'une maladie chronique est un bon révélateur de cette exploration que tentent les dispositifs de formation en s'aventurant dans des situations problématiques, jusqu'ici chasses gardées des thérapeutes, pour que ces situations soient traitées aussi en termes d'acquisition de connaissances et de compétences de préférence au seul soin qui risque de maintenir l'adulte dans un état de dépendance, au lieu de l'ouvrir vers d'avantage d'autonomie (Lacroix, Assal 2011).

Il n'empêche qu'on ne saurait traiter de la même façon, d'un côté une formation promotionnelle destinée à conférer plus d'autonomie au stagiaire adulte, de par l'acquisition qu'il va devoir faire de nouvelles compétences et/ou de nouvelles connaissances, de l'autre une formation destinée à sortir le stagiaire adulte de l'état de dépendance dans lequel il se trouve par la mise en place de nouveau repères, le cas échéant de nouveaux savoir-faire. Si les méthodologies peuvent être variées dans le premier cas, pour le second, ces méthodologies auront impérativement à prendre en compte trois paramètres structurant des situations d'apprentissage en contexte de vulnérabilité : 
- premier paramètre structurant, la constitution d'un collectif de travail formé du groupe de stagiaires, cooptés ou non mais soucieux de développer entre eux des liens de coopération au travers des communauté d'apprentissage (Cristol 2016) ;

- deuxième paramètre structurant, l'explicitation du but visé par la formation proposée, un but si possible négocié entre le formateur et le groupe de stagiaires ; cette explicitation d'un but négocié est au centre des pédagogies du projet, qui en ont fait leur facteur de réussite, à condition toutefois que ce but aboutisse à un résultat matérialisable et communicable (Le Grain 1985) ;

- troisième paramètre structurant, une pédagogie concrète portant sur des situations-problèmes, sur des éléments situationnels familiers mais questionnant pour les stagiaires (Michel 2001).

Sans doute, au-delà de cette dichotomie formation promotionnelle/formation réparatrice, pourrait-on trouver d'autres logiques de formation ayant pignon sur rue ; il s'agirait alors d'en évaluer leur pertinence au regard du contexte dans lequel elles sont utilisées; les formations tant occupationnelles que conjoncturelles que par ailleurs les formation-action relèvent de ces autres logiques pour ce qui concerne les formations non validantes car il est évident que les instances de formation ayant une finalité de validation sont d'une autre nature car elles nous font entrer dans un univers plus contraignant laissant moins de liberté pour la constitution du collectif, pour la négociation et pour la mise en place d'une pédagogie concrète.

\section{Par la formation, l'autonomie adulte change actuellement de sens}

Si l'adulte étalon évoqué plus haut était pris dans un tissu de repères d'où il lui était difficile de s'échapper et qui avait tendance à constituer un cadre de vie normé, l'adulte en maturation que nous avons encore appelé en perspective appartient à la génération qui le suit et va vouloir s'échapper de ce cadre, considéré comme trop enfermant pour conquérir son autonomie et aspirer à la liberté. Cette revendication d'autonomie caractéristique de cette génération d'adultes en perspective comportait une double dimension existentielle et culturelle: à l'époque il s'agissait d'apprendre à vivre hors des sentiers convenus de la transmission intergénérationnelle et en même temps il était question pour les jeunes générations d'alors de se doter des modes de vie plus individualistes initiées par la société de consommation. C'est sans doute la raison pour laquelle une telle revendication d'autonomie est peu redevable à l'une ou l'autre formation alors existante. On peut même affirmer qu'elle s'est faite hors champ de la formation ; c'est là reconnaître que la formation continue ne travaille que secondairement sur l'émancipation ; son souci premier, mais en cela elle se distingue de la formation initiale, c'est de permettre au stagiaire 
adulte d'accomplir ce mouvement paradoxal d'autonomisation engendrant un double changement de posture :

- $1^{\mathrm{er}}$ changement: déformation ou déconstruction, déstructuration par rapport aux savoirs et savoir-faire, savoirs expérientiels acquis antérieurement, mis à distance ;

- 2ème changement: reformation ou reconstruction, restructuration de ces savoirs et savoir-faire, articulés avec de nouveaux savoirs et savoir-faire, savoirs expérientiels sur un mode moins approximatif, plus soucieux des exigences du réel et de ses résistances.

L'adulte en maturation s'est donc trouvé dans cette conjoncture historique et culturelle, sans doute exceptionnelle, celle des années 1960-1970 où, hors champ de la formation, il a été amené, pour le meilleur ou pour le pire, à conquérir son autonomie pour mieux exister dans sa liberté d'initiative, donc à s'émanciper des contraintes et codifications jugées par lui désuètes, celles dont a hérité l'adulte mature et que ce dernier veut lui transmettre. Cet adulte en perspective réalisait ainsi ce vœu tout fait moderniste que déjà, à la fin de sa vie qui était la fin du XVIII siècle triomphant, formulait E. Kant en 1784 à travers son fameux Sapere aude : Ose te servir de ton entendement, sois libre ${ }^{3}$.

Cette première forme d'autonomisation, l'autonomie-émancipation qui perdure encore aujourd'hui mais sous une forme beaucoup plus discrète et minoritaire va être rattrapée voire même anéantie par une seconde forme d'autonomisation, celle-là beaucoup plus récente car très actuelle et, elle, en grande partie véhiculée par le champ de la formation : il s'agit de l'autonomie-maintien comme norme qui doit assurer à l'adulte son indépendance coûte que coûte malgré ou à cause de l'état de vulnérabilité dans lequel il se trouve dans le contexte socioculturel actuel (Jouan, Laugier 2009). Ce contexte en effet est devenu celui d'un Etat-providence qui a dû abandonner ses prérogatives, faute de moyens suffisants, les situations de prises en charge pour raison de dépendance coûtant cher dans leur prise en charge par cet Etat : financement du chômage pour les personnes privées d'emploi et incapables d'en trouver un, financement des soins pour les malades, financement des institutions pour personnes âgées dépendantes, financement du revenu minimum d'insertion pour les personnes sans travail depuis longtemps... Il s'agit donc dans un tel contexte d'identifier pour les édicter au sein de formations appropriées, les règles à prendre en compte, soit pour aider les personnes dépendantes à devenir autonomes, soit pour donner les moyens aux personnes autonomes de conforter leur autonomie. Qu'elle soit promotionnelle ou réparatrice, peu importe, la formation aujourd'hui doit pour la collectivité viser, même si c'est par des voies différentes, le même but : faire sortir l'adulte d'un état de dépendance qui le contraint ou le menace, un état coûteux à différents points de vue et conforter l'autonomie par une formation ou un accompagnement de maintien de façon à ce

${ }^{3}$ In : Réponse à la question : Qu'est-ce que Les Lumières? 
que cet adulte ne soit plus à la charge des institutions ou le moins possible : ainsi en est-il de cette autonomie des assistés pour utiliser la formulation forgée par Nicolas Duvoux (2009), en opposition à l'autonomie comme émancipation, ce qu'Alain Ehrenberg (1995) reformule dans le contraste entre l'autonomie comme valeur et l'autonomie comme norme : peu importe les mots s'ils entendent exprimer la même idée!

\section{Face à la montée des individualisations, l'accompagnement comme substitut de la formation}

Nous constatons depuis deux ou trois décennies à une montée en puissance des dispositifs individualisants, la culture de l'info com et du numérique aidant. Cette montée de l'individualisation va d'autant plus toucher les dispositifs de formation, que ceux-ci dans leur version réparatrice se montrent ou sont perçus comme insuffisamment efficaces, au regard de la diminution des précarités ambiantes et de la réduction du taux de chômage, ce dernier étant bien souvent considéré par les medias comme la seule référence à prendre en compte. Alors à partir des années 1990, pour la rendre plus efficace, on va chercher institutionnellement à individualiser la formation et cette individualisation va se concrétiser, à dix années d'intervalle (1991-2002) dans la mise en place de deux dispositifs de formation individualisée originaux qui vont connaître leur heure de gloire, les bilans de compétences personnelles et professionnelles en 1991, les validations des acquis de l'expérience en 2002. Ces deux dispositifs vont nécessiter pour être mis en œuvre des accompagnements appropriés de l'adulte-candidat pour qu'il puisse effectuer son bilan de compétences, ou structurer ses acquis de l'expérience : on voit là, avec quelques années de recul comment bilans de compétence et V.A.E. vont être les principaux opérateurs d'une mutation de la formation en accompagnement (Boutinet et al. 2007).

Mais parler d'accompagnement, qu'est-ce à dire? Il semble tout d'abord opportun de faire remarquer que c'est à partir des années 1990, pour en rester au contexte français, que nous assistons à ce développement spectaculaire des pratiques d'accompagnement dans une diversité de domaines professionnels en même temps que nous observons les actions de formation opérer un repli en volume et en demande. Ces accompagnements sont surtout individuels, confortant cette tendance à l'individualisation de nos sociétés, évoquée un peu plus haut. Les accompagnements de groupe existent certes mais apparaissent peu fréquents. Il est donc loisible de considérer l'accompagnement individuel comme une variante de formation individualisée. Aussi restons-en à cet accompagnement individuel qui désormais accompagne tous les âges de la vie, de l'accompagnement de la parturiente à l'accompagnement de la personne souffrante en fin de vie. Quel que soit le type d'accompagnement en cause, il manifeste une belle transversalité institution- 
nelle, faisant fi des contraintes économiques dans la mesure où il peut se dérouler tout aussi bien dans le cadre d'une rémunération professionnelle de l'accompagnateur/trice que dans celui du simple bénévolat.

Ce qui caractérise une pratique d'accompagnement c'est son double caractère structurant, relationnel et directionnel. Comme l'a bien mis en évidence Maela Paul (2004), accompagner consiste à assumer simultanément deux postures acceptées et intériorisées par chacun des protagonistes, personne accompagnée et accompagnateur/trice : être avec et aller vers. Alors que la formation reste souvent trop inféodée aux contenus à transmettre, l'accompagnement ne comporte pas de contenu, sinon secondairement; il est d'abord organisé autour d'un invariant à double face : sortir l'autre de sa solitude et se donner du temps pour aller avec lui vers un but à définir ensemble. Dans la dynamique relationnelle de l'accompagnement deux projets ont à se dire et à se questionner, celui de la personne accompagnée d'abord et en écho celui de l'accompagnateur/trice ; ces deux projets gagnent à en faire advenir un troisième, le projet partagé, projet d'accompagnement porté par les deux protagonistes.

Finalement les pratiques d'accompagnement se fondent sur deux interdits, ne pas laisser seule la personne en demande d'accompagnement, ne pas rester immobile avec elle et par ces deux interdits nous retrouvons l'exigence sociale à laquelle ils répondent, que nous avons présentée plus haut : redonner à l'adulte son autonomie, voire préserver cette dernière, voire encore la développer ; car, quel que soit son degré d'autonomie, la personne accompagnée est en situation de précarité, blessée par la vie, au travail, en famille, dans l'espace associatif ou ailleurs (Ehrenberg 1995) et l'accompagnement en singularisant une relation dans le être avec, en suscitant une action à réaliser par le aller vers va faire œuvre formatrice mais davantage sur le mode du préceptorat que sur celui du groupe de stagiaires en formation.

\section{Place de l'informel dans la formation : le parcours atypique de l'adulte transitionnel}

La formation pour adultes est connue par ses aspects formels et encadrée la plupart du temps selon les circonstances par les paramètres suivants: une commande institutionnelle, un stage, un(e) ou des formateurs/trices patenté(s), un diagnostic initial pour tester les besoins ou demandes de formation des stagiaires, une évaluation terminale. Elle se manifeste, nous venons de le voir, aussi mais à travers des aspects moins formels dans l'un ou l'autre mode d'accompagnement individualisé. Plus discrète et insaisissable, elle existe néanmoins tout au long de la vie quotidienne de l'adulte, mais là, de façon non formelle au cours de son expérience personnelle ou professionnelle; elle peut surgir aussi de façon tout à fait informelle à travers l'irruption d'un évènement qui surprend dont l'imprévu est porteur 
d'apprentissage. Nous apprenons de notre expérience, comme nous apprenons de l'inédit évènementiel mais à condition de temps à autre de revenir sur cette expérience ou sur cet évènement pour en capitaliser les acquis identifiables.

Si par commodité de langage dans ce qui va suivre, nous regroupons dans le même ensemble informel, le non formel et l'informel que nous venons d'évoquer et que les années 2000 par l'intermédiaire de l'UNESCO ont cherché à distinguer, nous constatons alors que cet informel chez certains adultes joue un rôle essentiel dans la conquête et la préservation de leur autonomie aux deux sens que nous avons donné plus haut à ce terme. Ces adultes dans leur retour verbalisé sur leur expérience ou sur des évènements qu'ils ont été amenés à vivre, manifestent au-delà des vulnérabilités et déstabilisations qui ont pu les affecter dans des situations inconfortables, une autonomie de maintien que N. Duvoux (2009) dénomme l'autonomie des assistés souvent doublée d'une autonomie d'émancipation.

Cette formation informelle par l'expérience ou par l'évènement peut se révéler de différentes façons : sans doute que l'histoire de vie a été et reste un lieu privilégié pour le faire mais dans l'énonciation des expériences, ou dans le partage d'expériences à travers des cadres prédéfinis, il y a dans les pratiques des histoires de vie souvent retour vers un certain formalisme de la formation Christine Delory-Momberger (2000). Restons donc délibérément dans l'informel tel qu'il va se manifester chez l'un ou l'autre adulte, qui au détour d'une conversation, glisse spontanément à propos de son itinéraire professionnel ou personnel, sous le mode de la confidence ou sous celui de la révélation, voire celui de l'intuition: "vous savez, moi, j'ai eu un parcours atypique!». Le temps disponible aidant, si cet adulte se laisse interroger pour qu'il dise en quoi il trouve son parcours atypique, il va commenter les grandes lignes de ce parcours avec ses moments clés de bascules, de transitions, de rebonds, découvrant en parlant, ce qu'il a appris de son expérience, pour peu qu'il puisse la verbaliser, laissant entendre que le fait de reconnaître lui-même l'atypicité de son parcours lui permet de se positionner dans la différence par rapport à son entourage. Cette atypicité confessée est pour lui un bon compromis existentiel : les blessures qu'inévitablement cette atypicité invoquée a engendrées sont compensées par une revendication identitaire permettant de mieux exister à travers ces deux marqueurs de reconnaissance que sont : se sentir différent, faire reconnaître son originalité.

Ainsi l'informel reste un bon exutoire pour cicatriser les vulnérabilités qui se sont installées au fil des évènements. Souvent il cohabite sans problème avec le formel de la formation. Nous venons d'appréhender l'informel du parcours atypique, de plus en plus souvent rencontré ; nous pouvons aussi évoquer une autre figure de l'informel qui semble présentement prendre de la place, la rupture intentionnelle de carrière: rompre de soi-même une situation professionnelle certes enviable mais qui n'apporte plus ce que l'on attendait d'elle car cette situation ne donne plus satisfaction en même temps qu'elle amène une crise du sens. Le rebond délibéré mais osé, ce rebond à faire ou déjà fait en direction d'une situation profes- 
sionnelle estimée moins enviable, moins prestigieuse mais plus jugée signifiante s'accompagne donc de recherches de compensations, tel le souci de pouvoir disposer d'une meilleure qualité de vie, d'une plus grande liberté de mouvement, du choix d'une activité qui fasse sens. De telles compensations momentanément vont permettre de donner subjectivement le change à la rupture, laquelle va progressivement constituer par la suite un nœud d'apprentissages existentiels, objet de réflexion formative.

Reconnaissance de l'atypicité de son parcours, rupture intentionnelle de carrière participent de la diversité de ces pratiques caractéristiques d'une démarche d'auto-formation au sein de laquelle l'informel et le formel de la formation s'entrecroisent continuellement. Une telle démarche d'autoformation qui depuis son émergence dans les années 1970 met au centre du dispositif de formation, l'adulte apprenant (Carré 1997) manifeste, comme l'indique Albert Bandura ([1995] 2002), l'agentivité de cet adulte, acteur social, soucieux de ne pas choisir entre les deux formes d'autonomie qu'il entend développer, l'autonomie comme valeur à perspective émancipatrice et l'autonomie comme norme, ordonnée au maintien.

\section{Un double défi reste à relever : un espace déqualifié, des temporalités exacerbées}

Les formations, lorsqu'elles sont formelles tout spécialement, à l'ère postmoderne du numérique et de l'accélération sont confrontées à un double défi à relever : comment peuvent-elles s'appuyer sur des espaces résilients et des temporalités structurantes?

Nos espaces sont en effet de plus en plus déqualifiés, relégués aux accessoires de l'uniformité et de la plasturgie. Tout est fait pour qu'ils se laissent modeler à dessein, quitte à ce que l'urbanisme les reproduise en série. Mais ce qui devient essentiel, c'est qu'ils cessent de résister lorsque l'on tente de les aménager. Ils plient sous les exigences du dernier effet de mode ou du dernier avatar technique. Certes on pourra trouver des exceptions à une telle évolution tendancielle : ainsi actuellement les paysagistes font porter leur attention depuis une ou deux générations sur les espaces ouverts comme lieux de rencontre entre nature et culture (Berque 1995) qu'il s'agit d'aménager en paysage par la médiation d'un projet de paysage initiateur. Par ailleurs la substitution voici une quarantaine d'années des pratiques urbanistiques de réhabilitation urbaine à celles de la rénovation urbaine a redonné vie à bon nombre de centre-ville historiques et cette entreprise de réhabilitation s'est avéré la plupart du temps une réussite dans la façon de mieux faire cohabiter la double requalification d'un espace à la fois urbain et historique, une requalification porteuse d'apprentissages informels pour les usagers de cet espace. Mais que dire des quartiers suburbains, pour la plupart toujours relégués ; 
que dire des zones périphériques d'aménagement concerté en bordure des centres bourgs ruraux, dont les constructions témoignent d'une grande banalité et d'une belle uniformité de façade pourvoyeuse d'ennui, engendrant ce que Marc Augé (1992) appelle un non-lieu?

De l'espace et spécialement de l'espace habité, qui en est une variante incontournable, nous semblons oublier qu'il est fait d'une articulation de vides et de pleins. Or une formation, par la force des choses, se déploie toujours dans un espace habité et c'est là qu'elle prend sens ou au contraire qu'elle se laisse dépréciée par un agencement problématique des vides et des pleins qui l'environnent. Cette formation ne saurait donc se penser en dehors de l'espace qui l'organise et fait à sa manière alterner les vides et les pleins, mais des vides et des pleins structurants ou au contraire laissés en déshérence, car en fonction du mode d'articulation réalisé entre ces vides et ces pleins, nous aurons tantôt des espaces créateurs vivifiant, tantôt des non-lieux.

Ce qui caractérise l'espace, c'est son hétérogénéité à travers la variété des éléments qui le constituent, qui peuvent offrir des capacités de résistance, nous faisant découvrir par derrière la réalité qui sollicite, le réel qui fait écran et résiste (Maldiney 1991) ; cet espace hétérogène se présente donc comme altérité mais en même temps et paradoxalement il est accueil au travers des relations significatives qu'il offre à nouer avec l'un ou l'autre de ses composants humains, animaux, végétaux, géologiques, techniques, au travers aussi des opportunités qu'il va pouvoir offrir pour peu qu'on le sollicite. Hétérogénéité et altérité d'un côté, relations significatives et opportunités de l'autre sont là pour le moins quatre paramètres spatiaux qui nourrissent les projets de formation ébauchés par les adultes si du moins les espaces au sein desquels ces derniers évoluent se montrent suffisamment stimulants dans leurs variétés de vides et de pleins.

Laissons maintenant de côté l'espace et venons-en au temps ou plus précisément aux temporalités qui se montrent à l'ère postmoderne comme de plus en plus exacerbées et invasives, du moins les temporalités dominantes qui organisent à ce jour nos vies quotidiennes ; ces dernières ont muté par rapport à ce qu'elles étaient à l'ère moderne ; ces nouvelles temporalités dominantes sont souvent imposées par l'usage intensif des technologies de l'information et de la communication et les exigences de la culture du numérique qui leur sont associées ; elles se caractérisent surtout par leur présentisme et leur évanescence (Hartog 2003) : temporalités de l'évènement et de l'évènementiel ${ }^{4}$, de l'immédiateté, de l'instantanéité et de l'urgence : ainsi dans l'urgence, comme dans l'immédiateté, il est bien difficile sinon impossible de déployer une démarche de formation; à travers l'évènement on le peut, en opposition en cela à l'évènementiel, mais à la seule condition d'utiliser les temporalités de récupération qui suivent l'évènement, ce qui n'est pas possible

\footnotetext{
${ }^{4}$ L'évènement dans son instantanéité et son imprévisibilité me surprend car il arrive malgré moi mais je fais advenir l'évènementiel désiré en organisant moi-même un évènement de courte durée destiné à être remplacé par un autre évènementiel qui va effacer le premier.
} 
pour l'évènementiel, toujours en interférence, de par son activisme volontariste avec un autre évènementiel.

De ce que nous venons d'évoquer, il appert que les pratiques de formation, pour éviter de se laisser abusées et défigurées, gagneraient à se faire pour une part contre-culturelles dans le choix qu'elles opèrent des temporalités ad hoc. Il leur faut renoncer, autant que faire se peut aux temporalités évanescentes pour privilégier des temporalités plus consistantes du court-terme, donc moins aléatoires, notamment celles de la transition et de l'alternance, appropriées pour toute action de formation. L'instance de formation est en effet à considérer comme un temps de transition précédé et suivi par deux alternances. Il est de ce point de vue intéressant de constater que la plupart des pratiques de formation, depuis maintenant un demi-siècle ont eu recours à ces deux temporalités qu'elles ont contribué largement à réhabiliter pour l'ère actuelle ; transition et alternance étaient en effet des temporalités disqualifiées en régime de modernité encore en vogue dans les années 1950-1960, car contrevenant aux temporalités dominantes de l'époque qui privilégiaient au contraire celles de la linéarité progressiste et que l'on retrouve dans l'idée de planification.

\section{Conforter les pratiques de formation, oui mais à quelles condi- tions?}

Plusieurs conséquences peuvent être tirées de ce qui précède ; sans vouloir constitue un trop large inventaire des possibles à notre disposition, nous nous limiterons ici à 4 propositions répondant aux défis que nous avons mis en évidence plus haut et qui interpellent les pratiques de formation:

- Redonner toute leur importance aux apprentissages informels qui se déploient tout au long d'un parcours de vie personnelle et/ou professionnelle pour les valoriser dans un environnement trop souvent marqué par l'inconfort de la vulnérabilité et de la précarité ; pour ce faire il est indispensable d'instaurer des collectifs de rencontres (séminaires, colloques, communautés d'apprenants, rencontres festives ou de travail) au cours desquels les adultes, en revenant sur leur parcours, vont avoir la possibilité, de verbaliser et conscientiser certains de leurs acquis d'expérience spontanément évoqués en grand groupe ou de préférence en groupe restreint ;

- Requalifier les espaces-temps des formations formelles en les réaménageant dans le souci de mettre à disposition des stagiaires des lieux d'accueil significatifs et propices à une ouverture sur une pédagogie de l'espace ; cette pédagogie aura la préoccupation d'organiser des temps susceptibles de privilégier le transitionnel par rapport à l'immédiat, un transitionnel situé paradoxalement à distance mais en articulation avec l'expérience passée, ce 
transitionnel ouvrant en cours de formation sur un horizon de possibles, par le biais d'alternances à saisir ou à aménager ;

- En partant du principe que toute formation est éclairée par un projet qui la modélise et l'oriente, permettre à ce projet de donner toute son importance à la recherche de singularité qui est l'une de ses propriétés fondatrices. Cette importance dévolue à la singularité interdit de faire un projet de formation clefs en main. Elle implique pour le/la ou les formateurs/trices d'accorder toute son attention à la prise en compte des deux singularités fondatrices de toute action de formation: la singularité de la situation au sein de laquelle se déploie la dite formation et la singularité du groupe de stagiaires. Cette double singularité nécessite de pousser le plus loin possible le travail de négociation formateur/trice-stagiaires concernant un aménagement approprié de la situation de formation ;

- S'assurer dans une démarche de formation d'un minimum requis de présentiel, notamment dans les dispositifs d'autoformation, dans les e-learning, dans les formations à distance ou dans les mooc. Le présentiel du groupe constitué par la dynamique des pairs et du formateur ou de la formatrice est structurant, permettant une co-formation authentique que ne saurait assurer aussi bien le réseau dans les formations à distance ou en ligne ; même si ces formations à distance ont des effets facilitateurs pour les échanges communicationnels rencontres qu'elles rendent possibles, elles ne sauraient remplacer le présentiel de la rencontre.

Cette énumération de propositions ne prétend pas à l'exhaustivité ; elle se veut du moins significative de ce que pourraient apporter présentement les dispositifs de formation en éléments structurants des parcours de vie mais de ce qu'ils ne font pas suffisamment dans les temps actuels.

\section{Que dire de la quête de sens chez l'adulte qui se forme?}

Le développement spectaculaire des technologies au cours des vingt ou trente dernières années a changé nos vies quotidiennes en leur apportant un confort insoupçonné et pourtant, sans doute rarement comme aujourd'hui nous sommesnous sentis autant précarisés et fragilisés dans ces vies quotidiennes par une violence latente devenue invasive qui manifeste une impossibilité à faire cohabiter ce confort issu du développement technique avec une capacité à exister sans cesse remise en cause à travers une privation subite de travail, la venue d'une maladie gravissime, un déficit de reconnaissance, l'un ou l'autre divorce avec les relations qui nous sont le plus proches. Cet inventaire de nos fragilités ressenties pourrait être prolongé encore longtemps pour pointer cette fracture progrès technique/régression sociale que beaucoup de nos contemporains viennent à évoquer spontanément en posant la question du sens de ce qu'ils vivent, de ce qu'ils font, du 
monde qui les entoure et par-delà cette question en apportant leur propre réponse sous l'angle de la perte de sens, de la montée de l'absurde : ce que nous vivons, ce que nous faisons, ça n'a pas de sens!

Nous touchons là à une question de philosophie existentielle de grande ampleur que nous laissons de côté ou plutôt limiterons à la question du sens dans le champ de la formation. Certes une telle question ouvre sur un espace projectif dans lequel chacun y met son trop plein de subjectivité. Mais en même temps, pour une personne, en l'occurrence ici un adulte, le fait de donner du sens à une activité, ici de formation garantit l'implication de cette personne par le lien d'intelligibilité mais aussi de sensibilité qu'elle instaure entre elle-même et l'activité qu'elle réalise ou l'action dans laquelle elle désire s'engager.

L'adulte qui se forme peut être considéré ou se considère lui-même en reconfiguration de sens pour donner une nouvelle interprétation à son existence, aux actions qu'il initie, comme le soulignait Jack Mezirow (2001). Cette reconfiguration dans le champ de la formation, nous proposons de la décliner à travers les 5 attributs suivants que l'on confère habituellement au sens :

- le sens-orientation que l'adulte entend donner à sa formation, dans le moment présent mais aussi pour le court et le moyen terme à travers telle ou telle priorité associée à la dite formation, tel ou tel projet qu'il entend réaliser grâce à cette formation ou tel objectif qu'il cherche à atteindre ;

- le sens-signification que l'adulte cherche à verbaliser pour préciser ce qui le motive à se former c'est à dire les justifications ou les raisons qu'il peut donner à son départ en formation, qui vont conférer au sens-orientation la légitimité subjective dont il a besoin ;

- le sens-sensorialité dans le contact signifiant que l'adulte noue avec l'espace qui l'environne et qui se trouve associé à la dite formation, l'espace de formation proprement dit ou son espace professionnel ou son espace personnel ;

- le sens-sensibilité dans la continuité ou la rupture que l'adulte entrevoit entre la formation présente et son passé de par un lien de familiarité ressenti à moins qu'il ne s'agisse à la place du lien d'une mise à distance choisie, voire d'une rupture entre ce qu'il cherche présentement et ce qu'il était ou faisait auparavant ;

- le sens-socialité dans le lien social de coopération que l'adulte va pouvoir développer avec ses pairs en formation comme il peut les développer, grâce à la formation suivie, avec les acteurs de son environnement de vie, un senssocialité qui interdit d'assimiler une formation à une variante d'ermitage.

Cette déclinaison du sens par les sens se montre sous sa face paradoxale, par laquelle le singulier de sens ne saurait être appréhendé que par l'intermédiaire de son pluriel. Certes une telle déclinaison n'implique pas que l'adulte, dans une obsession totalisante, convoque tous les sens à la fois mais au moins plusieurs d'entre eux, deux voire trois, car toute recherche de sens, si elle se refuse 
à l'exhaustivité de tous les sens possibles, ne saurait se laisser enfermer dans l'exclusivité d'un seul sens : loin de l'absolu, elle revendique la relativité. Ces deux extrêmes de l'exclusivité et de l'exhaustivité conduisent en effet plus à une posture idéologique totalitaire qu'à une ouverture questionnante, celle du sens interrogé par un possible horizon absurde, par un non-sens éventuel ou probable : non-sens et absurde nous placent face à des situation-limite qui constamment questionnent ce sens conféré à nos entreprises dont l'adulte réflexif ne peut faire l'économie. Opter pour cette perspective de relativité et d'ouverture du sens dans le champ de la formation, c'est ici reconnaître que cette dernière ne saurait avoir réponse à tout ; elle n'a pas le dernier mot.

\section{Références bibliographiques}

Augé M. (1992) Non-lieux, une introduction à une anthropologie de la sur-modernité, Paris, Le Seuil.

Bandura A. ([1995] 2002) Auto-efficacité, le sentiment d'efficacité personnelle, Bruxelles, de Boeck.

Berque A. (1995) Les raisons du paysage, Paris, Hazan.

Boulte P. (1995) Individus en friche, essai sur l'exclusion, Paris, Desclée de Brouwer.

Boutinet J.-P. (1999) L'immaturité de la vie adulte, Paris, Presses Universitaire de France.

Boutinet J.-P. et al. (2007) Penser l'accompagnement adulte, ruptures, transitions, rebonds, Paris, Presses Universitaire de France.

Boutinet J.-P. (2013) Psychologie de la vie adulte, Paris, Presses Universitaire de France.

Bujold Ch., Gingras M. (2000) Choix professionnel et développement de carrière, Montréal, Gaëtan-Morin.

Carré Ph. (1997) Trois approches de l'autoformation in : Ph. Carré, A. Moisan, D. Poisson, L'autoformation, Paris, Presses Universitaire de France : 1-40.

Cristol D. (2016) Les communautés d'apprentissage : apprendre ensemble, « Savoirs... » (à paraître).

Delory-Momberger C. (2000) Les histoires de vie, de l'invention de soi au projet de formation, Paris, Anthropos.

Dubar Cl. (1985) La formation professionnelle continue, Paris, La Découverte. 
Duvoux N. (2009) L'autonomie des assistés, Paris, Presses Universitaire de France.

Ehreberg A. (1995) L'individu incertain, Paris, Calmann-Lévy.

Hartog F. (2003) Régimes d'historicité, présentisme et expériences du temps, Paris, Seuil.

Holland J.-L. (1973) Making vocational Choices, a theory of careers, Prentice Hall Englewood Cliffs.

Houde R. (1999) Le temps de la vie, Montréal, Gaétan Morin.

Jouan M., Laugier S. (2009) Comment penser l'autonomie, entre compétences et dépendance, Paris, Presses Universitaire de France.

Lahire B. (1998) L'home pluriel, Paris, Nathan.

Lacroix A., Assal J.-P. (2011) L'éducation thérapeutique des patients, Paris, Maloine.

Lapassade G. (1963) L'entrée dans la vie, essai sur l'inachèvement de l'homme, Paris, Editions de Minuit.

Le Grain (1985) Le défi pédagogique, Bruxelles, Vie ouvrière.

Maldiney H. (1991) Penser l'homme et la folie, Grenoble, Millon.

Mezirow J. (2001) Penser son expérience, Lyon, Chronique sociale de Lyon.

Michel J. (2001) La formation des adultes, Québec, Les Editions logiques.

Osterrieth P. (1966) Faire des adultes, Bruxelles, Dessart.

Paul M. (2004) L'accompagnement, une posture professionnelle spécifique, Paris, L'harmattan.

Rogers C. (1961) On becoming a person, Boston, Houghton Mifflin.

Jean-Pierre Boutinet

\section{Kształcenie dorosłych - narzędzia konceptualne}

W ciągu kilku ostatnich dziesięcioleci kształcenie dorosłych we Francji stopniowo rozwijało własną terminologię, odmienną od terminologii stosowanej w kształceniu ogólnym, wprowadzając pojęcia: dorosły, stażysta, kształtują- 
cy, doświadczenie, kompetencja, refleksyjność czy działanie, aktor, odpowiedzialność, uznanie. Pojęcia te odzwierciedlają istotę kształcenia dorosłych, które kształtowało się na przestrzeni ostatnich dziesięcioleci XX w. Specyfikę tę wyznaczają oczywiście także inne pojęcia: praktyka, przebieg/ścieżka, przekształcenie, kształcenie $\mathrm{w}$ trakcie pracy zawodowej, a także analiza sytuacji. Wszystkie te pojęcia dalekie są od pojęć: uczeń, nauczyciel, program, pedagogika, ocenianie, kontrola, które są podstawowymi pojęciami w kształceniu ogólnym. Jednakże kształcenie ustawiczne, którego celem był awans społeczny dorosłych, napotyka na poważny problem z chwilą pojawienia się, począwszy od 1975 r., bezrobocia strukturalnego, które dotknęło dużą część społeczeństwa. Zjawisko to doprowadziło do konieczności ukierunkowania lub przeorientowania wielu form kształcenia ustawicznego na inne cele umożliwiające włączenie społeczne poprzez znalezienie zatrudnienia czy powrót na rynek pracy.

Tak więc kształcenie nastawione na awans zawodowy, na które było zapotrzebowanie w latach 1950, 1960, 1970, w szczególności w społeczeństwie francuskim, napotyka na poważne trudności. Celem tego kształcenia było z jednej strony dostosowanie się do postępu technicznego, a z drugiej osiąganie go poprzez kształcenie, gdyż jedno i drugie miało zapewnić społeczeństwu nowe optymistyczne perspektywy na przyszłość. Jednakże w ciągu dwu ostatnich dekad ubiegłego wieku okazało się, że kształcenie dorosłych $\mathrm{z}$ trudem radzi sobie w sytuacji braku stabilizacji zawodowej dorosłych, w coraz większym stopniu bezbronnych wobec koniunktury ekonomicznej, z początku bardzo obiecującej, która gwałtownie spadała, a stan ten utrzymywał się przez długie lata. Koniunktura ta miała wpływ tak na sferę pracy, podlegającą ciągłej transformacji, jak i na sferę zatrudnienia, coraz trudniejszego do zdobycia, w kontekście nawracających kryzysów, które naznaczyły ostatnie 40 lat.

W niniejszym tekście podejmujemy próbę odpowiedzi na dwa pytania: jakimi narzędziami konceptualnymi należałoby posłużyć się w kształceniu ustawicznym w myśleniu o trudnej przyszłości dorosłych w obecnym kontekście postmodernistycznym? Jaki sens powinien przyjąć termin: „przyszłość dorosłych", którym posługuje się kształcenie ustawiczne, aby móc ominąć skutki trwającego kryzysu, dyskredytującego wszelką perspektywę budowania przyszłości?

Tłumaczenie: Grażyna Karbowska 